\title{
Impact of Gulfstream-IV Dropsondes on Tropical Cyclone Prediction in a Regional OSSE System
}

\author{
KELly Ryan, LiSA BUCCI, AND JAVIER DELGADO \\ NOAA/AOML/Hurricane Research Division, and Cooperative Institute for Marine and \\ Atmospheric Studies, University of Miami, Miami, Florida \\ ROBERT ATLAS \\ NOAA/Atlantic Oceanographic and Meteorological Laboratory, Miami, Florida \\ SHIRLEY MURILLO \\ NOAA/AOML/Hurricane Research Division, Miami, Florida
}

(Manuscript received 3 May 2018, in final form 4 January 2019)

\begin{abstract}
Aircraft reconnaissance missions remain the primary means of collecting direct measurements of marine atmospheric conditions affecting tropical cyclone formation and evolution. The National Hurricane Center tasks the NOAA G-IV aircraft to sample environmental conditions that may impact the development of a tropical cyclone threatening to make landfall in the United States or its territories. These aircraft data are assimilated into deterministic models and used to produce real-time analyses and forecasts for a given tropical cyclone. Existing targeting techniques aim to optimize the use of reconnaissance observations and partially rely on regions of highest uncertainty in the Global Ensemble Forecast System. Evaluating the potential impact of various trade-offs in the targeting process is valuable for determining the ideal aircraft flight track for a prospective mission. AOML's Hurricane Research Division has developed a system for performing regional observing system simulation experiments (OSSEs) to assess the potential impact of proposed observing systems on hurricane track and intensity forecasting. This study focuses on improving existing targeting methods by investigating the impact of proposed aircraft observing system designs through various sensitivity studies. G-IV dropsonde retrievals were simulated from a regional nature run, covering the life cycle of a rapidly intensifying Atlantic hurricane. Results from sensitivity studies provide insight into improvements for real-time operational synoptic surveillance targeting for hurricanes and tropical storms, where dropsondes released closer to the vortex-environment interface provide the largest impact on the track forecast. All dropsonde configurations provide a positive 2-day impact on intensity forecasts by improving the environmental conditions known to impact tropical cyclone intensity.
\end{abstract}

\section{Introduction}

Improvements in prediction on any scale require both an accurate representation of the atmospheric conditions occurring at a given time and accurate model behavior to estimate conditions at subsequent times. Marine atmospheric environments can be difficult to forecast because of the sparse measurements obtained in these regions. Aircraft reconnaissance missions are vital in gathering direct atmospheric mass and

Corresponding author: Kelly Ryan, kelly.ryan@noaa.gov momentum measurements over the ocean that satellites are unable to collect. NOAA conducted experiments between 1982 and 1996 using their WP-3D Hurricane Hunter aircraft (P-3) that allowed for the collection of inner-core and near-storm environment observations using omega-based dropwindsondes (Burpee et al. 1996). These data provided a significant improvement in tropical cyclone (TC) track guidance issued by the National Hurricane Center (NHC) and suggested that direct measurements had the potential to significantly reduce forecast errors (Burpee et al. 1996). Synoptic surveillance missions using a Gulfstream-IV (G-IV) jet aircraft and 
new GPS dropwindsondes (dropsondes) began during the 1997 hurricane season in an effort by NOAA's National Hurricane Center (NHC) and Atlantic Oceanographic and Meteorological Laboratory/Hurricane Research Division (HRD) to improve track forecasts of potentially damaging tropical cyclones. Despite improvements seen in P-3 dropsonde collection in the 1980s to early 1990s, impacts using G-IV dropsonde have been marginal at best (Aberson 2002; Majumdar et al. 2013; Aberson and Franklin 1999) and are limited to only short-term forecast improvements. Optimizing the collection and use of such data is paramount due to limited aircraft and dropsonde resources.

Targeting methods have been adopted that refine the data collection procedure in TCs and their surrounding environments. The ability to predict optimal locations prior to a deployment depends on assumptions regarding five factors: the probability of large forecast errors in a high-impact event, the influence of routine observations, the projected influence of targeted observations, characteristics of the data assimilation (DA) scheme and characteristics of the observation type (Majumdar et al. 2011). Variability in results among different targeting techniques remains an issue in determining the best approach for targeting tropical cyclones. Subjective targeting schemes, such as the deep-layer mean (DLM) wind variance guidance, focus on sampling the synoptic-scale upper-level wind, which is known to affect TC track (Aberson 2003). For recurving Atlantic hurricanes, singular vector (SV) approaches pinpoint initial state sensitivity in the upstream trough and near-storm environment regions (Peng and Reynolds 2006). However, highly sensitive regions that are identified depend on the field used to define uncertainty (Reynolds et al. 2007). Techniques using the adjoint-derived sensitivity steering vector (ADSSV) relate sensitive regions at the sampling time to steering flow at the verifying time (Liou and Chou 2008). This technique identifies regions that affect the steering flow in the vicinity of a given $\mathrm{TC}(\mathrm{Wu}$ et al. 2009). In contrast to these methods, ensemble transform Kalman filter (ETKF) guidance suggests sampling in areas downstream of the midlatitude storm track in addition to adjacent synoptic-scale features (Majumdar et al. 2011). This ensemble-based method estimates the predicted error variance associated with a given feature by determining error growth estimates from an analysis (Bishop and Toth 1999; Bishop et al. 2001; Torn and Hakim 2008, 2009; Xie et al. 2013; Torn 2014).

Adaptive, probabilistic sensitivity techniques such as total energy singular vector (TESV) and ETKF guidance have shown that no method is superior in every scenario (Sellwood et al. 2008). Major differences among techniques appear when stratifying by TC strength. In a comparison study by Majumdar et al. (2006), TESV and ETKF identify similar regions in only $30 \%$ of cases for weaker systems, and in only $20 \%$ of the cases do TESV and DLM guidance select the same regions. Emerging targeting methods that account for data assimilation schemes show promise, such as adjoint-based techniques (Daescu and Todling 2010) and Hessian singular vectors associated with 4Dvariational DA, although few have been implemented. Observing system experiments (OSEs) have proven valuable in assessing various targeting methods, as is demonstrated in (Aberson et al. 2011). Although the sample size is small, their results suggest that the three techniques evaluated are able to determine where extra dropsondes will impact the track forecast the most.

Many of the above techniques were considered to define NHC's G-IV targeting procedure for tasked synoptic surveillance sampling. However, no technique has proven significantly beneficial for all hurricanes. Current software operates by using regularly spaced dropsonde observations in regions of large forecast spread in the NCEP bred-vector ensemble forecasting system and has shown a $25 \%$ improvement in the 2-day track forecast using the variance in DLM wind (Aberson 2003). The purpose of this study is to investigate potential alterations to the current operational procedure.

Observing system simulation experiments (OSSEs; Atlas 1997) offer a cost-effective way to evaluate tradeoffs in observing system design and are ideal for testing various targeting techniques. Like OSEs, the objective is to assess the potential impact of observing systems on analyses and forecasts. Evaluating data using OSSEs has the added advantage of allowing new designs to be assessed without the need for aircraft resources. Results from OSSEs can be used to inform the operational community by utilizing key components of the operational HWRF model. AOML's regional hurricane OSSE system provides such a framework for performing sensitivity tests necessary to optimize G-IV targeting (Atlas et al. 2015a). Previous projects using this OSSE framework quantify the impacts of data on hurricane forecasting and include the assessment of various space-based observing systems such as the Optical Autocovariance Wind lidar (Atlas et al. 2015b), CYGNSS (McNoldy et al. 2017), and hyperspectral sounders (Ryan et al. 2016). A similar approach will be presented here using airborne-derived observations. This study goes beyond the track and intensity forecast error analysis completed in the above experiments by examining the meteorological fields that affect hurricane track and intensity prediction. The analysis focuses on evaluating the potential impact of the current G-IV 


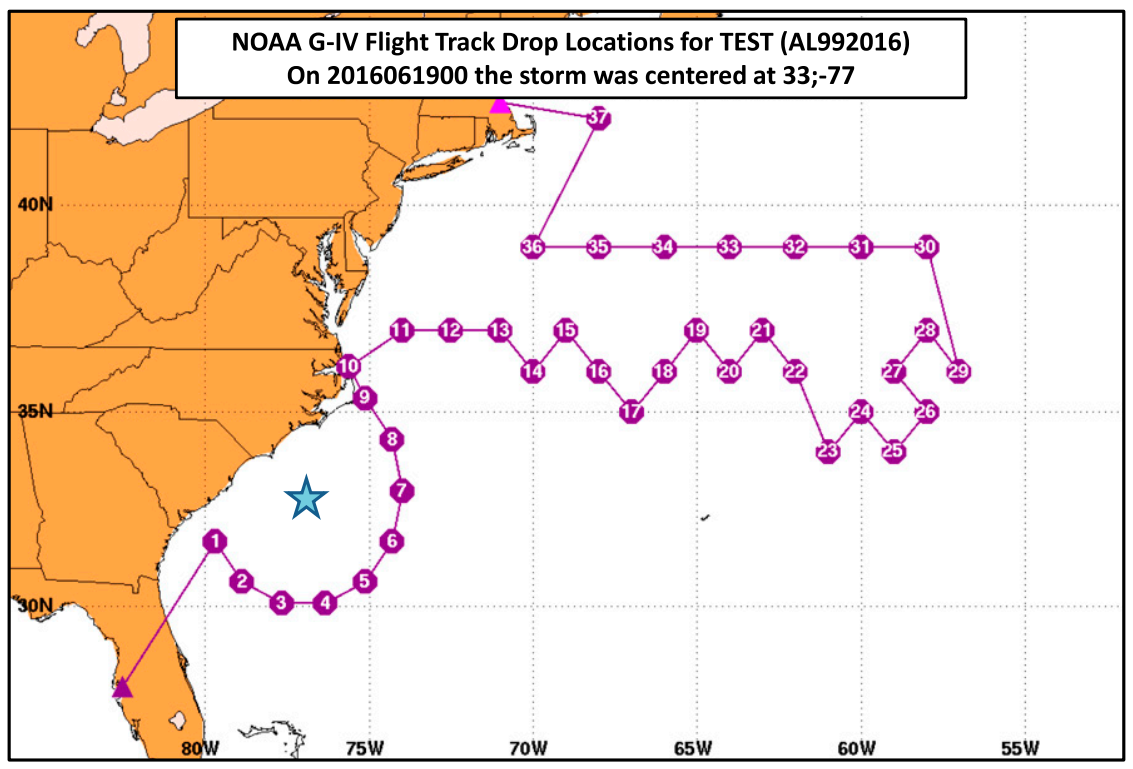

FIG. 1. Example output from NHC's targeting software. The purple line represents the G-IV flight track, purple dots indicate dropsonde locations, and the blue star defines the "TEST" hurricane position.

targeting procedure and investigates potential changes to the circumnavigation flight track where dropsondes would be deployed in the near-storm environment.

The rest of this paper is outlined as follows. Section 2 describes the specifics of NHC's current operational G-IV targeting procedure, and section 3 provides a detailed overview of AOML's hurricane regional OSSE system. Section 4 contains four subsections that describe the control experiment, initial testing of the current targeting method, storm-relative radial dropsonde location experiments, and storm-relative azimuthal dropsonde location experiments. Conclusions from the experiments are summarized in section 5 .

\section{Targeting methodology}

The National Hurricane Center tasks NOAA's G-IV aircraft when a TC threatens the United States or its territories. Each tasked mission is determined 2-3 days prior to an expected landfall and occurs repeatedly in 12-h intervals. Mission designs are based on a technique developed nearly 20 years ago which aims to target the most uncertain regions of the environment affecting a given TC (Aberson 2003). Current surveillance missions sample the near-TC environment and the downstream synoptic-scale features. NHC's targeting software determines the locations where the G-IV will deploy dropsondes in both of these two regions. This two-part flight track includes dropsonde locations every $1^{\circ}-2^{\circ}$ designated by 1 ) areas of highest variance in GEFS deep-layer mean wind within $20^{\circ}$ of the TC and 2) a circumnavigation at a radius of $2^{\circ}-3^{\circ}$ from the TC center. The result of using this methodology provides NOAA's Aircraft Operations Center (AOC) with one flight track as shown in Fig. 1. Although the current methodology is partially automated, initial parameters, such as dropsonde distribution, must be defined by the forecaster on duty.

To optimize this type of targeting, sensitivity experiments must be broken up by region and controlled for changes to each part of the flight pattern. Dropsonde spacing should be tested in both the near-storm and downstream environments. Since AOML's regional hurricane OSSE system (discussed in section 3) uses

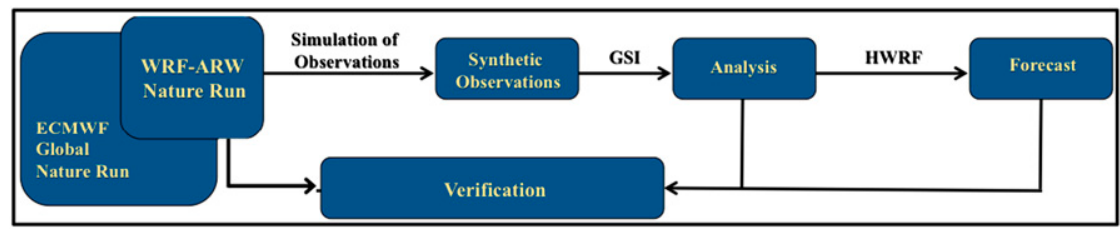

FIG. 2. Framework of AOML's regional hurricane OSSE system. 
(a)

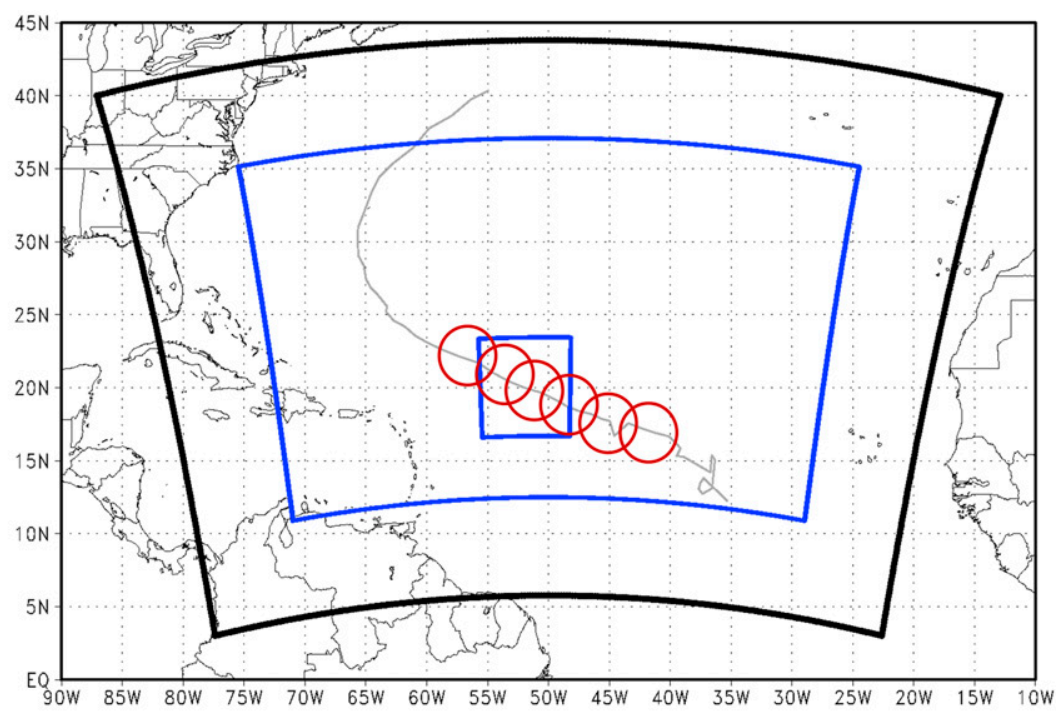

(b)

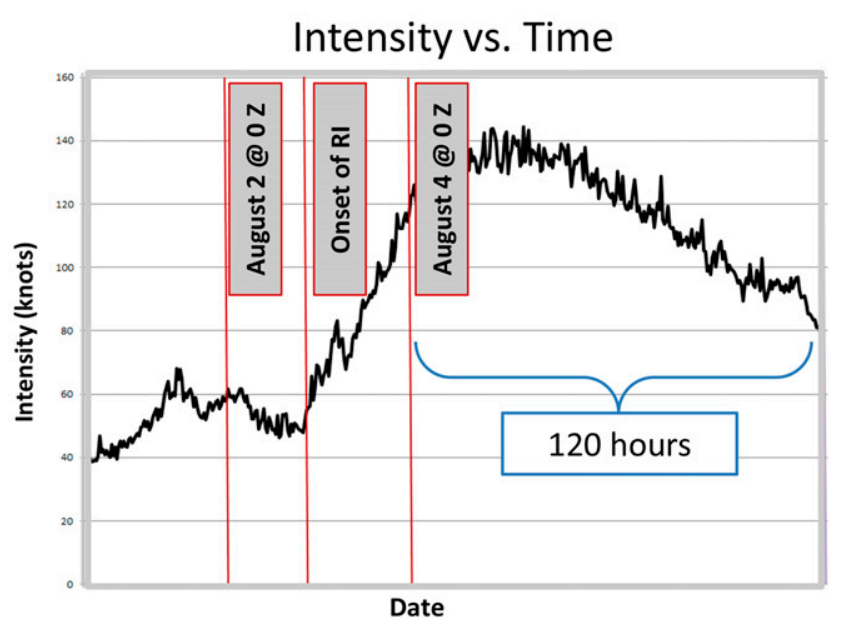

FIG. 3. (a) Domain configuration for AOML's regional hurricane OSSE system. The domain outlined in black defines the hurricane nature run (HNR) parent domain. Blue boundaries define the parent $(9 \mathrm{~km})$ and nested $(3 \mathrm{~km})$ domains of the modified HWRF Model. The gray line specifies the HNR storm track, and the red circles represent the flight paths of a simulated G-IV aircraft for one experiment. (b) Intensity evolution of HNR. Red lines indicate the start and end cycle dates and the onset of rapid intensification (RI). The intensity plot ends at the date of the 120-h forecast from the last assimilation cycle.

HWRF to evaluate this data, sensitivity tests examined in this paper focus on the storm-relative location of dropsondes in the near-TC environment. Uncertainty in alternate variable fields from GEFS should also be optimized, but this would require the use of ensemble-based data assimilation on a large domain such as GFS and are therefore beyond the scope of the current study.

\section{OSSE methodology}

The framework of AOML's regional hurricane OSSE system is displayed in Fig. 2 and depicts a series of steps required to conduct each individual experiment. Assessing all of these steps is vital to ensure that results describe real-world impacts. A standardized approach to evaluating each of these steps is provided by Hoffman and Atlas (2016).

A long-term global model simulation of the atmosphere is typically used as a nature run (NR) and acts as the truth in OSSEs. For TCs, a much higher-resolution NR is necessary to capture smaller-scale features that the global NR cannot represent. For this reason, a high-resolution WRFARW hurricane nature run (Nolan et al. 2013) was embedded within the lower-resolution ECMWF T511 global nature run (Reale et al. 2007). This hurricane nature run 
TABLE 1. Conventional observations assimilated in these experiments, measuring temperature $T$, specific humidity $q$, instrument pressure $P$, station pressure $P_{\mathrm{stn}}$, and zonal and meridional winds $(u, v)$.

\begin{tabular}{|c|c|c|}
\hline Observing network & Details & Observation type \\
\hline Aircraft & ACARS, MDCRS, reconnaissance (RECON) flight level & $T, q,(u, v)$ \\
\hline Aircraft & AIREP, PIREP, AMDAR, communication and navigation (CAN) & $T,(u, v)$ \\
\hline Rawindsonde & Profile & $T, q,(u, v), P_{\mathrm{stn}}$ \\
\hline Dropsonde & Profile, splash & $T, q,(u, v), P$ \\
\hline Pibal & Profile & $(u, v)$ \\
\hline Surface marine reports & Surface & $T, q,(u, v), P_{\mathrm{stn}}$ \\
\hline Surface land reports & Surface & $T, q, P_{\mathrm{stn}}$ \\
\hline NOAA profiler network & Profile & $(u, v)$ \\
\hline Satellite wind reports & Cloud top & $(u, v)$ \\
\hline WindSat reports & Scatterometer & $(u, v)$ \\
\hline Marine ASCAT winds & Scatterometer & $(u, v)$ \\
\hline NEXRAD VAD & Radar & $(u, v)$ \\
\hline
\end{tabular}

(HNR) spans the 13-day life cycle of a rapidly intensifying North Atlantic TC and utilizes a fixed $27-\mathrm{km}$ resolution parent domain with three vortex-following nests of resolutions 9,3 , and $1 \mathrm{~km}$, respectively. It is essential that the HNR in these experiments is of higher resolution than the forecast model. This preserves the assumption that the forecast model is not capable of capturing all existing scales of motion in reality.

To evaluate different observation configurations, G-IV aircraft paths were simulated from the aforementioned HNR as shown in Fig. 3a. Dropsonde data were simulated along these flight tracks using realistic trajectories. These dropsonde paths are horizontally advected by the wind and respond to vertical forcing from updrafts and downdrafts. The simulated temperature, moisture, and wind retrievals were collected at standard pressure levels to mimic the vertical resolution of dropsonde data assimilated in real-time operational models. Dropsonde measurement errors were given to each variable to reflect their respective errors in reality. These dropsonde data are assimilated along with standard control observations using the GSI 3D-variational (3D-Var) data assimilation (DA) scheme which performs analyses on the 9-km parent domain of HWRF (v3.5). The control data include TC vitals ${ }^{1}$ and the conventional and satellite observations listed in Tables 1 and 2, which reflect the operational data assimilation procedure (Tallapragada et al. 2015). These perfect simulated observations are generated from the global T-511 NR during the entire life cycle of HNR and do not consider the cloud field. The generation of satellite data is governed by the times and locations of measurements collected during the summertime of 2012. Simulated satellite observations were produced using the Community Radiative Transfer Model

\footnotetext{
${ }^{1} \mathrm{TC}$ vitals include the position and mean sea level pressure of the established TC vortex.
}

(CRTM) in conjunction with temperature and moisture fields from the NR (Zhu et al. 2012; Riishojgaard et al. 2012). One inherent limitation of the satellite data in this system is that radiances increase the domainwide errors due to bias correction and model top effects. Although this is inconsistent with global model results, regional models have shown difficulty when including these observations. The effects, however, are small when compared to the impact of observing systems and do not change the sensitivity of such experiments. The system performs a 6-h spinup and cycles every $6 \mathrm{~h}$, with 120 -h forecasts produced each cycle. Because of the limitations of the 3D-Var DA scheme which relies on a synoptic-scale climatological error covariance, this study focuses on the impact of these data on the near-TC environment. Forecasts are created on a 3-km vortex-following inner nest using a modified HWRF that omits vortex initialization/ relocation and ocean coupling, but is otherwise as described in Tallapragada et al. 2015. The domain configuration is displayed in Fig. 3a. This forecast model has 61 vertical levels and utilizes Ferrier microphysics, simplified Arakawa-Schubert cumulus parameterization, and the GFS planetary boundary layer and GFDL radiation schemes (Tallapragada et al. 2015). The difference in grid structure and parameterizations between the HNR and HWRF ensures that an identical twin ${ }^{2}$ experiment is avoided. Finally, a verification of the OSSE system is needed to relate potential impacts back to the real world. Tropical cyclone error trends in the OSSE system were compared to OSE error trends for an analog tropical cyclone. Using observation minus background errors for each cycle time, error trends are similar when comparing track and intensity forecast errors.

\footnotetext{
${ }^{2}$ Identical twin experiments are defined in OSSE literature as using the same model core and physics to develop the nature run as is used for generating forecasts (Atlas 1997).
} 
TABLE 2. Satellite observations assimilated in these experiments. GPS, global positioning system radio occultation; MHS, Microwave Humidity Sounder; GRAS, GNSS Receiver for Atmospheric Sounding; IASI, Infrared Atmospheric Sounding Interferometer.

\begin{tabular}{|c|c|c|c|}
\hline Satellite & Infrared & Microwave & GPSRO \\
\hline Aqua & AIRS & AMSU-A & - \\
\hline GOES-15 & sounder [SNDR-D(1-4)] & - & - \\
\hline$M e t O p-A$ & HIRS4, IASI & AMSU-A, MHS & GRAS \\
\hline NOAA-15 & - & AMSU-A & - \\
\hline NOAA-18 & - & AMSU-A, MHS & - \\
\hline NOAA-19 & HIRS4 & AMSU-A, MHS & - \\
\hline
\end{tabular}

It is important to note that these OSSEs make use of only one HNR representing one type of TC existing in nature. Thus, the results presented can only measure the potential impact of G-IV dropsonde data for a TC with characteristics such as the one represented by the HNR and not generalized for all TCs.

\section{Analysis: Sensitivity to storm-relative location}

The impact of G-IV dropsonde data is evaluated with respect to the suite of observations that are currently used operationally. All of the experiments are performed during the intensification period of the HNR between the times of 1200 UTC 1 August and 0000 UTC 4 August. The following analyses describe the relative impact of G-IV dropsonde data compared to a control experiment which represents existing operational capabilities in the regional OSSE system. These results focus on near-storm environment impacts that indicate realistic improvements using the synoptically driven 3D-Var DA system.
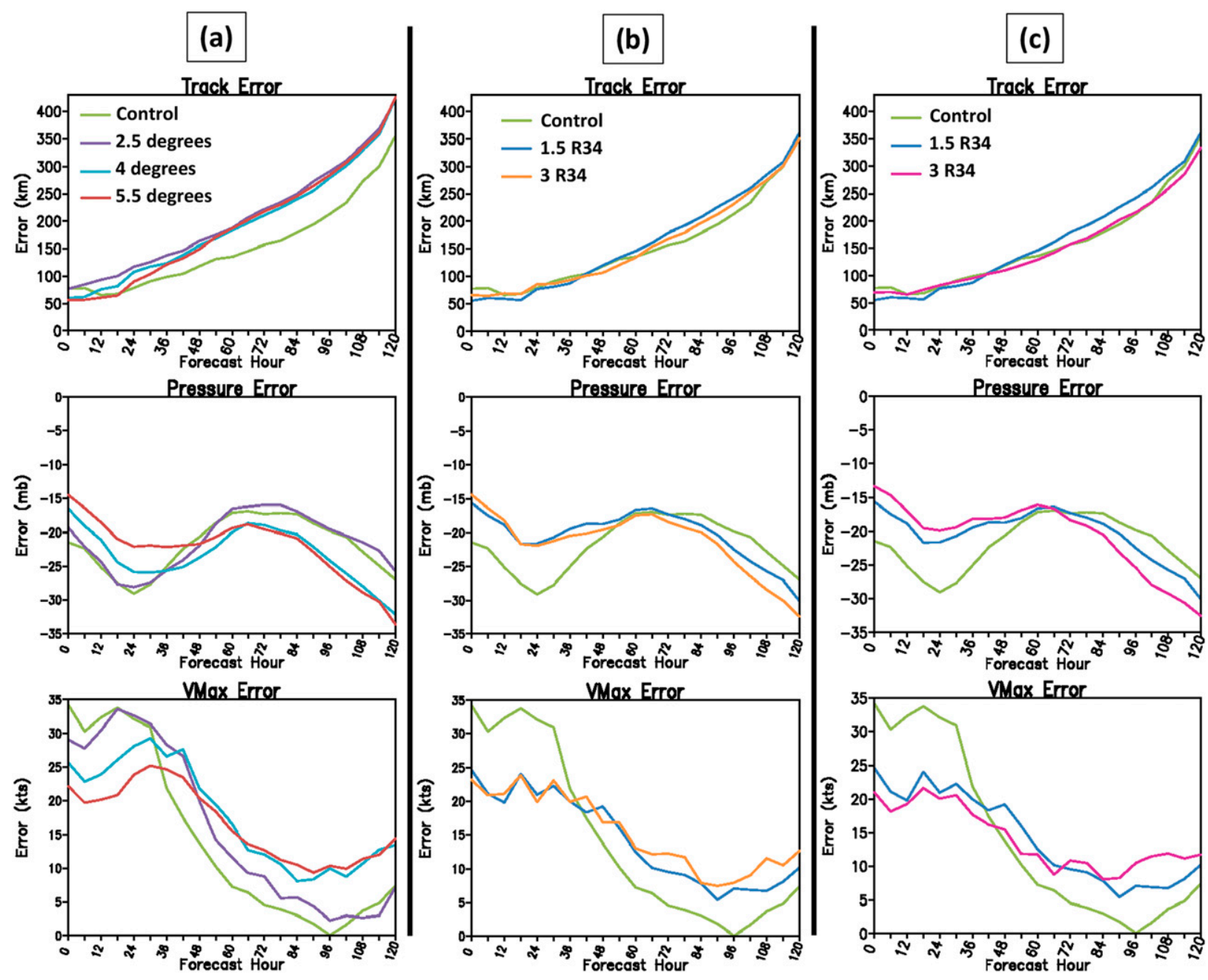

FIG. 4. Absolute track and intensity errors averaged over all 12 cycles for the following sets of experiments: (a) experiments testing radial distances, where control (green), $2.5^{\circ}$ (purple), $4^{\circ}$ (light blue), and $5.5^{\circ}$ (red) radial distances, (b) experiments testing the normalized radial distances, where control (green), 1.5 R34-unit (blue), and 3 R34-unit (orange) radial distances using $40^{\circ}$ storm-relative dropsonde spacing, and (c) experiments testing the dropsonde along-track resolution, where control (green), 1.5 R34-unit radial distance with $40^{\circ}$ storm-relative dropsonde spacing (blue), and 3 R34-unit radial distance with $20^{\circ}$ storm-relative dropsonde spacing (purple). 
TABLE 3. 10-m maximum wind (vmax) intensity and radius of galeforce (34 kt) winds for HNR displayed at 12 -h intervals.

\begin{tabular}{ccc}
\hline \hline Cycle time & $\begin{array}{c}\text { Vmax intensity } \\
(\mathrm{kt})\end{array}$ & $\begin{array}{c}\text { Radius of gale-force } \\
\text { winds (n mi; 1 n } \\
\text { mi = 1.852 km) }\end{array}$ \\
\hline 1200 UTC 1 Aug & 57 & 121 \\
0000 UTC 2 Aug & 56 & 106 \\
1200 UTC 2 Aug & 50 & 103 \\
0000 UTC 3 Aug & 77 & 135 \\
1200 UTC 3 Aug & 90 & 143 \\
0000 UTC 4 Aug & 123 & 143 \\
\hline
\end{tabular}

\section{a. Control experiment}

Assimilated data in the control experiment reflect the observations used to update the operational models in real time. These synthetic observations are simulated from ECMWF's T511 global nature run and include both satellite and conventional measurements described in (Tallapragada et al. 2015). Figure 4 displays the forecast track and intensity errors from the control experiment averaged over all 12 cycles. Errors are defined by the difference between the forecast values and HNR. Intensity is defined by the minimum sea level pressure (MSLP) and maximum 10-m winds (VMAX). Although the trend in track error ${ }^{3}$ appears to mirror track errors seen in operational prediction, intensity errors show a nonlinear evolution over time due to imbalanced initial conditions provided by 3D-Var analyses. These transient adjustments are enhanced at those cycles during which HNR is experiencing rapid intensification (RI, not shown). The stronger the hurricane, the farther the forecast error covariance is from the climatology-based background error covariance, thus requiring the DA system to adjust substantially in subsequent cycles and offsetting potential RI. The "spindown" issue prevalent in the modeling of TCs (Hendricks et al. 2013; Gopalakrishnan et al. 2012) likely contributes to this.

\section{b. Current targeting procedure and initial tests}

The data added to the control in the first comparison experiment replicates the circumnavigation flight patterns used in real-time G-IV targeting. Dropsondes are simulated every 1 earth-degree $(\approx 111 \mathrm{~km})$ along a G-IV flight track located 2.5 earth-degrees from the TC center. This is repeated for 6 missions occurring in 12-h increments as displayed in Fig. 3a. The time of interest focuses on the rapid intensification period of the HNR

\footnotetext{
${ }^{3}$ Track error is defined as great circle difference between HNR track and experiment track positions.
}

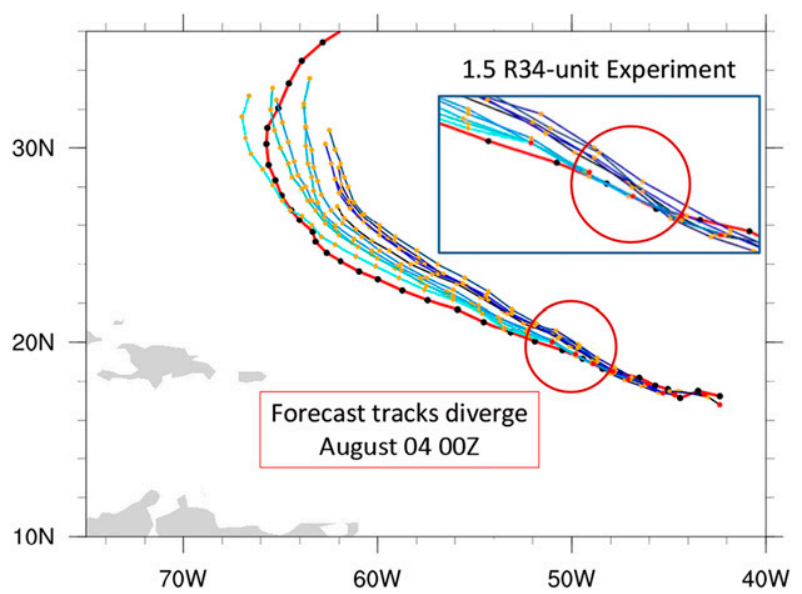

FIG. 5. Trend in track by cycle for the 1.5 R34-unit radial distance experiment. Earlier cycles starting 1800 UTC 1 Aug are dark blue and trend lighter for each subsequent cycle, where the final cycle at 0000 UTC 4 Aug is the lightest blue. A zoomed-in image is boxed for clarification. The red circle draws attention to the track divergence at 0000 UTC 4 Aug.

as indicated by Fig. 3b. In Fig. 4a, the dark purple line represents the error trends averaged over all 12 cycles when the data using current targeting procedures are assimilated. Although this data appears to degrade the long-term track forecast overall, there is a short-term improvement in intensity forecast skill. This phenomenon is investigated in the following experiments.

Initial radial sensitivity experiments use this same methodology for dropsonde spacing and incrementally change the G-IV distance from the TC center by $1 / 2^{\circ}$ for each experiment. Figure $4 \mathrm{a}$ shows the error trends for a subset of these experiments, depicting $2.5^{\circ}, 4^{\circ}$, and $5.5^{\circ}$ distances. In comparing these experiments, there is an increasing improvement in short-term intensity forecast skill as the G-IV deploys dropsondes farther from the center. A short-term improvement in track errors also occurs, but all experiments degrade the long-term track forecast. Further analysis reveals that these impacts may be due to the number of dropsondes deployed and how they constrain the extent of the TC wind field. Table 3 displays the evolution of gale-force wind radii for the nature run hurricane (HNR). The size of the TC changes throughout the period of interest, therefore changing the meteorological conditions that the simulated dropsondes measure at different assimilation cycles. Furthermore, the number of dropsondes changes when increasing the G-IV distance from the center. These two subtleties prevent consistent impacts from any one experiment and inhibit the ability to determine the sensitivity of changing the radial location of the G-IV aircraft. By choosing G-IV flight patterns relative to known TC characteristics, these subtleties are removed. 


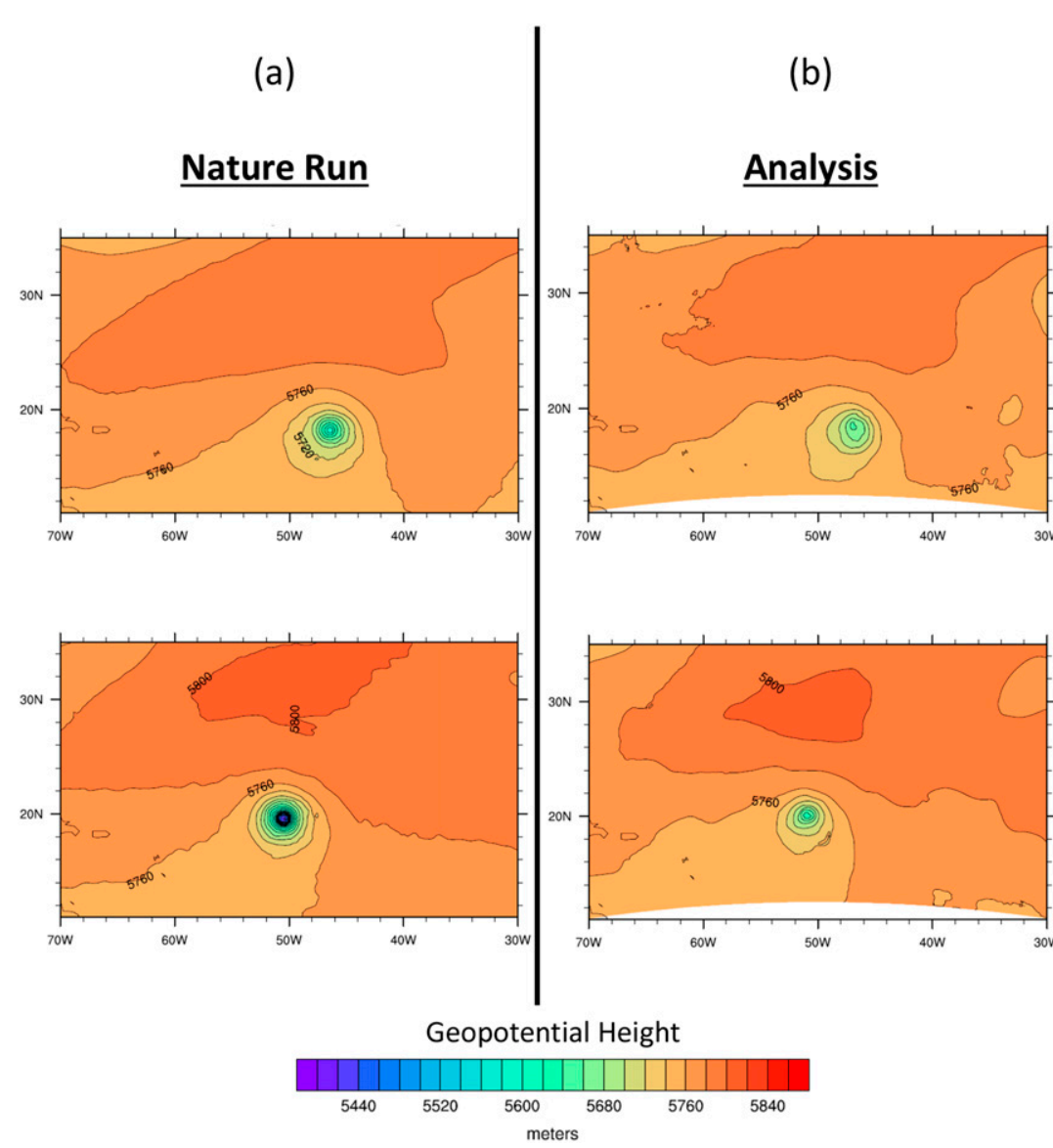

(c)
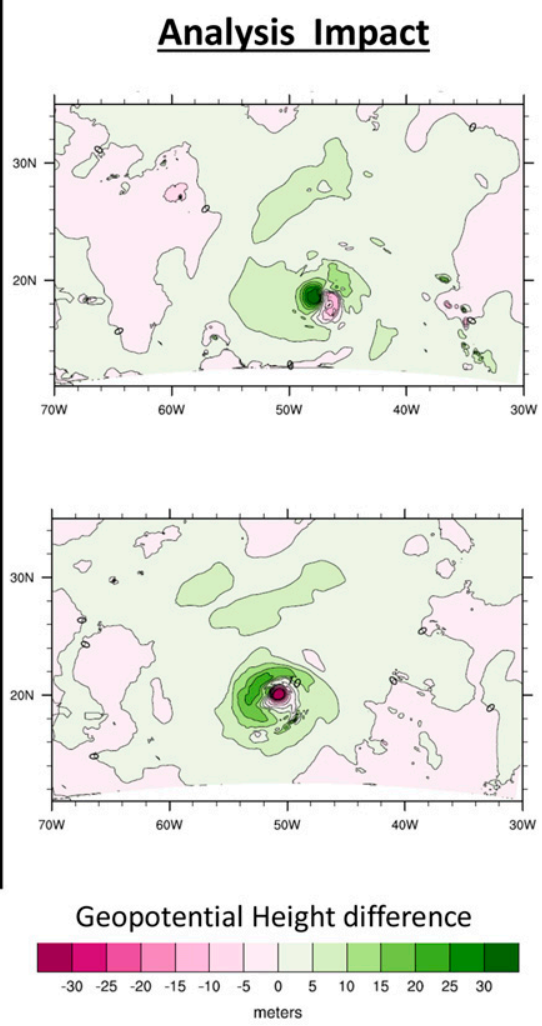

FIG. 6. (top) 500-mb geopotential height fields for 0000 UTC 3 Aug and (bottom) 0000 UTC 4 Aug for (a) HNR, (b) analysis fields for the 1.5 R34-unit radial distance experiment, and (c) impact of 1.5 R34-unit experiment observations on the 500-mb geopotential height analysis field compared to the control experiment. In (c) green colors indicate where the 1.5 R34-unit experiment is closer to HNR and pink colors indicate where the control experiment is closer to HNR.

In a new set of experiments, the G-IV distances for investigating the sensitivity to radial location of dropsondes are defined in terms of the radius of gale-force $\left(34 \mathrm{kt} ; 1 \mathrm{kt}=0.5144 \mathrm{~m} \mathrm{~s}^{-1}\right)$ winds. The dropsonde distribution is determined by azimuthal location relative to the TC center. Using this uniform configuration, new experiments are performed to study the sensitivity of TC prediction to dropsonde location.

\section{c. Storm-relative radial location}

Radial sensitivity experiments were revisited defining radial distance in terms of HNR size. The existing targeting procedure is represented by a G-IV flight track located at a radius of one R34 unit with a total of 10 dropsondes deployed at a spacing of $40^{\circ}$ azimuthally relative to the TC center during each simulated mission. The comparison radii tested range from one-half R34 units to three R34 units in increments of one-half R34 units. During each of these experiments, approximately 1000 synthetic observations are added in the assimilation.
Figure $4 \mathrm{~b}$ shows the track and intensity error statistics averaged over all cycles for the control experiment compared to 1.5 and 3 R34-unit aircraft radial distances which represent the two dominant trends in forecast error. Although a positive impact on intensity forecasts beyond $48 \mathrm{~h}$ is shown for both radii, there is little distinction between the two radial distances and neither experiment performs well during rapid intensification. The track forecast improves upon the control experiment out to almost two days when assimilating G-IV data near the radius of 34-kt winds. Experiments using a G-IV distance of $3 \mathrm{R} 34$ units provided a few short periods of improved track prediction but were less consistent then 1.5 R34-unit experiments. As was described in Zhang et al. (2011), errors for lead times longer than $36 \mathrm{~h}$ can be explained by the overfitting of observations in the 3D-Var scheme.

The 36-h positive impact of the $1.5 \mathrm{R} 34-$ unit experiment sheds light on the location where G-IV dropsondes may provide the most impact on track forecasts. Figure 5 
(a)

(b)

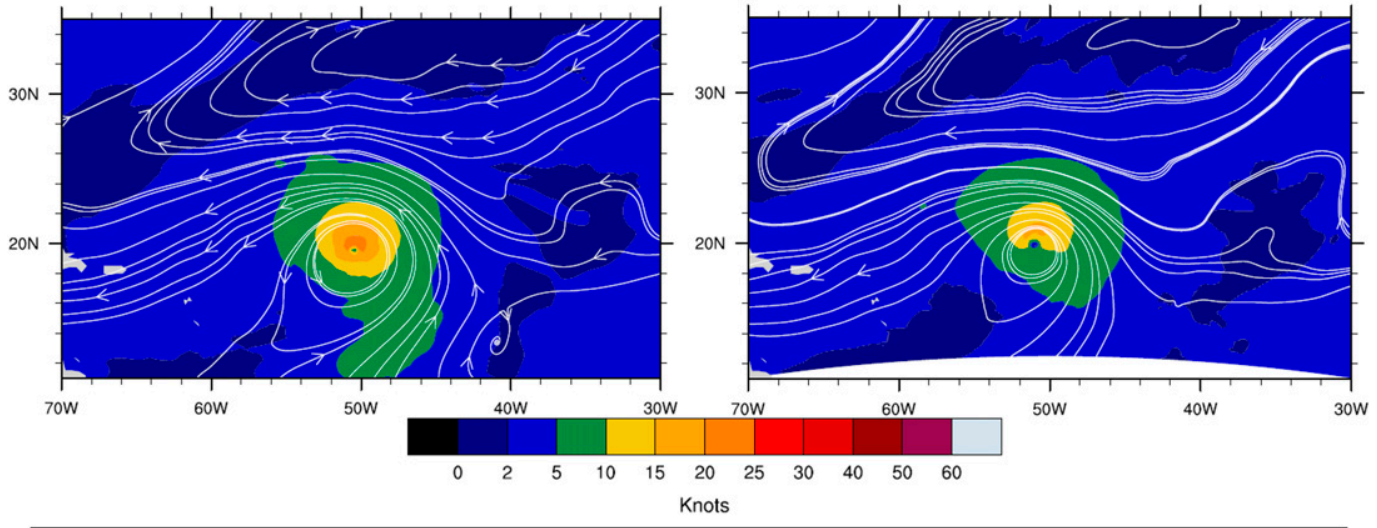

(c)

(d)

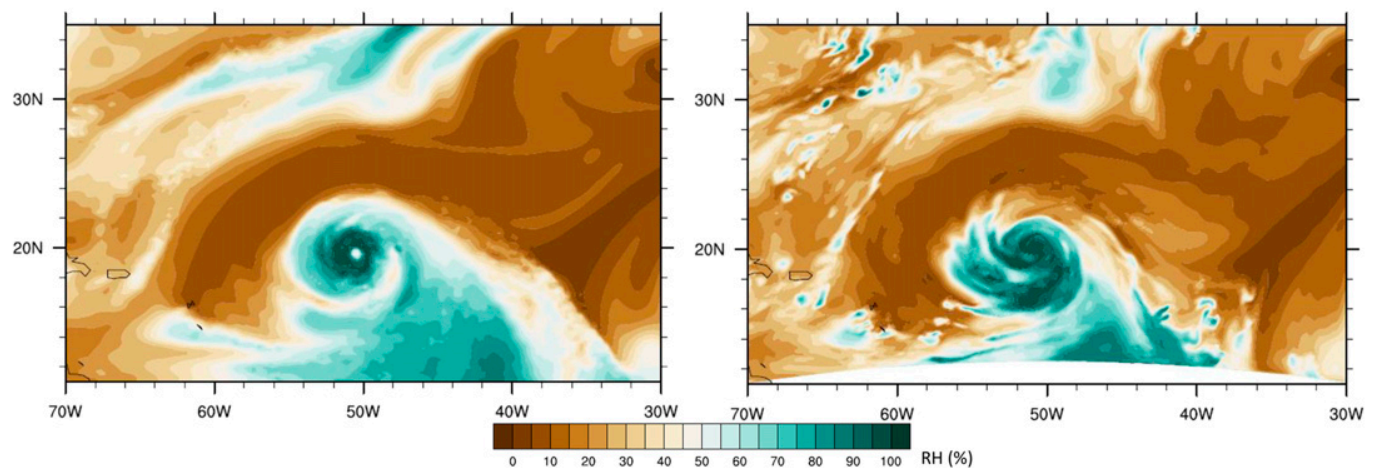

FIG. 7. (top) Deep-layer mean wind fields at 0000 UTC 4 Aug for (a) HNR and (b) the analysis produced by the 1.5 R34-unit experiment. (bottom) Midlevel relative humidity at 0000 UTC 4 Aug for (c) HNR and (d) the analysis produced by the $1.5 \mathrm{R} 34$-unit experiment.

shows the trend in track forecasts per cycle for the 1.5 R34-unit G-IV flight pattern. Forecast tracks diverge from each other around the 12th cycle (0000 UTC 4 August) where later cycles capture the HNR track more accurately. Figure 6 a features the nature run geopotential fields from 3 August (top) and 4 August (bottom) both at 0000 UTC. Figure $6 \mathrm{~b}$ displays the $500 \mathrm{mb}(1 \mathrm{mb}=$ $1 \mathrm{hPa}$ ) analysis fields at these times when assimilating data from this experiment. In the HNR, the vortex drifts along the outer edge of the high just after RI. The experiment, however, results in a ridge that dips farther south, allowing the vortex to embed itself within the high. The vortex is also substantially weaker and shallower, therefore limiting the force of large-scale impact on steering (Fig. 7a), where steering is defined by the deeplayer mean wind, a mass-weighted vertical average of winds in a column centered on each grid point. A depiction of the spatial impact of these data on the analysis geopotential fields is shown in Fig. 6c and confirms the improvement of nearby environmental conditions as indicated by widespread green coloring. The coupled nature of the impact field near the core is indicative of the shift in position of the vortex with the addition of these data. This limits the potential track improvements gained using these data and supports the average error trend comparison between experiments. These results provide further evidence to support the notion that TC location depends on anomalies in the Mid-Atlantic Ridge (Elsner et al. 2000; Evans et al. 1991; Bell and Chelliah 2006, etc.).

Figure $8 \mathrm{~b}$ shows the $48-\mathrm{h} 500 \mathrm{mb}$ geopotential height forecast fields initialized at 0000 UTC 3 August (top) and 0000 UTC 4 August (bottom) using data obtained from the 1.5 R34-unit experiment, revealing the difference in synoptic-scale flow given the position and strength of the subtropical ridge. The positive impact on track forecast before rapid intensification is cut short due to the lack of western extent of the ridge and differences in vortex size and strength. Forecast vortex differences in intensity and orientation after rapid intensification appear to be largely due to discrepancies in midlevel relative humidity approaching the western edge of the HNR location (Fig. 7c) where the core is 

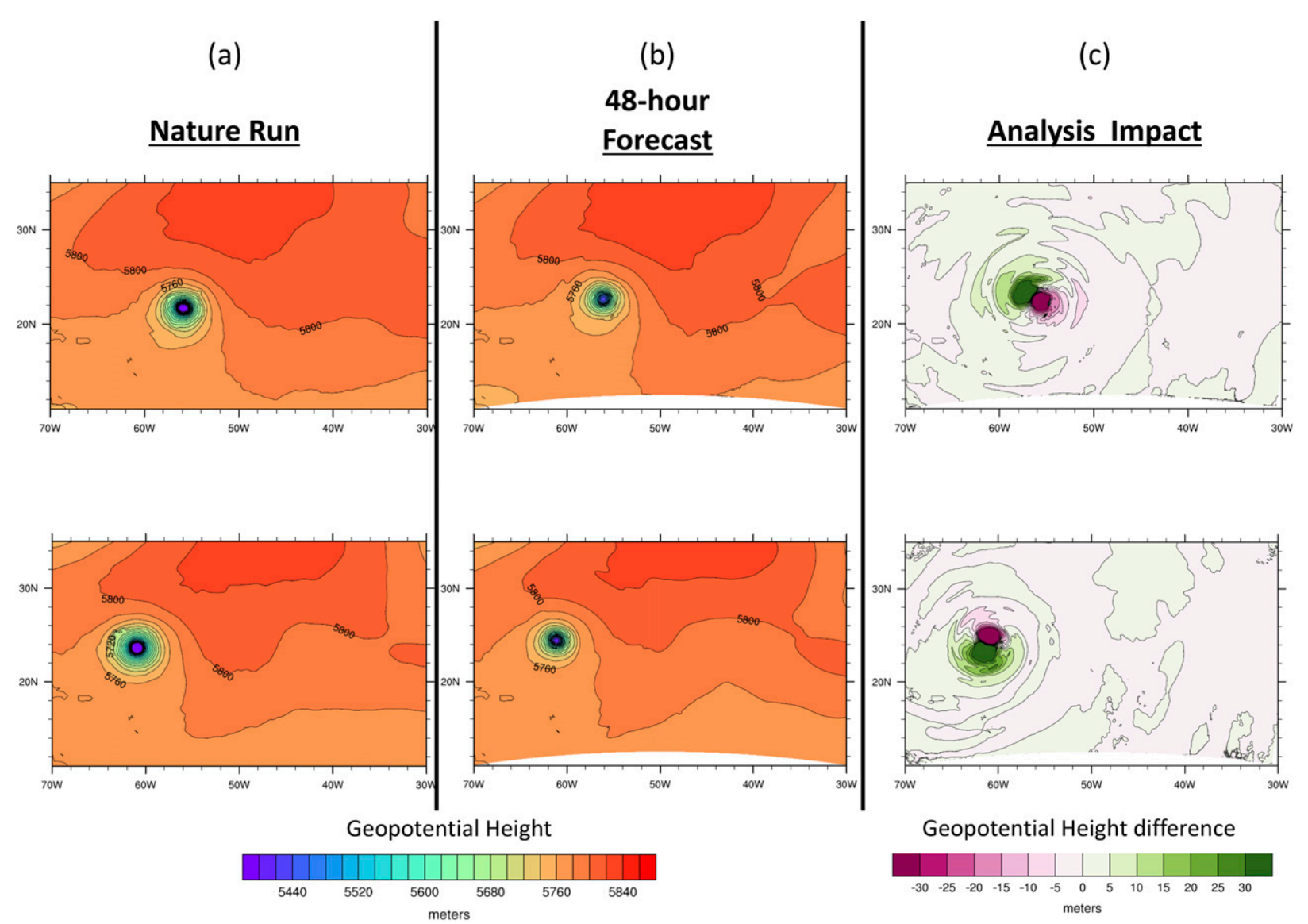

FIG. 8. As in Fig. 6, but for (top) 0000 UTC 5 Aug and (bottom) 0000 UTC 6 Aug of (a) HNR, (b) 48-h forecast fields, and (c) 48-h forecast impact compared to the control experiment.

protected by midlevel moisture, producing a smaller vortex, rather than entraining drier air into the circulation. The smaller, weaker vortex drifts until it is deep enough for the synoptic-scale flow to significantly impact its trajectory. This allows for improvement in the shortterm track forecast due to the lack of forcing by the larger scales and a degradation in the long-term track forecasts due to an incorrect environmental flow. This is reflected in the 48-h forecast geopotential height impact fields in Fig. 8c, where the majority of the impact is near the vortex and due to differences in the center position. Therefore, short-term track forecast improvements are due to an improved environment.

\section{d. Azimuthal location}

Azimuthal sensitivity experiments evaluate the distribution of G-IV dropsondes along a given circumnavigation flight track. Using the two radial distances described in the section above, various uniform dropsonde distributions were tested. Dropsonde locations are defined by azimuthal location relative to the TC center position and simulated at $20^{\circ}, 30^{\circ}, 40^{\circ}, 45^{\circ}$, and $60^{\circ}$ spacing.
About 100 synthetic observations per dropsonde are added in the assimilation. Figure $4 \mathrm{c}$ shows the track and intensity error statistics averaged over all cycles for the control experiment compared to the two best experiments: $40^{\circ}$ spacing for $1.5 \mathrm{R} 3$-unit radius and $20^{\circ}$ dropsonde spacing for $3 \mathrm{R} 34$-unit radius. Although the best short-term track impact is produced by the 1.5 R34-unit G-IV distance using dropsondes spaced $40^{\circ}$ apart, no significant differences in track prediction exist among the added experiments. This is reflected in the downwind environment fields which show little differences among experiments (not shown). In contrast to track forecast errors, the average intensity errors display a consistent improvement for over 2 days when assimilating dropsondes every $20^{\circ}$ at an aircraft distance of 3 R34 units from the center. Improvements to the vortex analyses are examined using near-TC environmental conditions known to affect the structure of TCs (DeMaria and Kaplan 1994, 1999; DeMaria et al. 2005; Elsberry et al. 1988; Landsea et al. 1998; Zhang and Sippel 2009, and others), such as entrainment of low-level equivalent potential temperature 
and midlevel moisture from the west and south. Based on the intensity forecast trends per cycle shown in Fig. 9, rapid intensification occurs more accurately throughout the later cycles despite the offset of timing. In other words, cycles initialized after the onset of RI perform better for track forecasts than those initialized prior to that time. For this reason, meteorological comparisons of the analyses focus on the last cycle.

Figures $10 \mathrm{~b}$ and 10c display the low-level (LL, $850 \mathrm{mb}$ ) equivalent potential temperature analysis fields for the two experiments on at 0000 UTC 4 August. Figure 10a shows the corresponding HNR field. Using dropsondes spaced every $40^{\circ}$ located at $1.5 \mathrm{R} 34$ units, cooler air is brought into the circulation from the south, and the core is small and much weaker than the HNR. Despite the weak vortex in the analysis for the comparison experiment with dropsondes every $20^{\circ}$ along the G-IV flight track at 3 R34-unit distance, the wind field is much broader and the higher temperature air is more consolidated near the center. Both experiments appear more saturated than HNR and share a similar near-TC environment profile (Fig. 11a), where near-TC environment extends $300-700 \mathrm{~km}$ outward in all directions. Largest differences in the near-TC environment exist south of the storm where although the LL profile for the 1.5 R34unit experiment better matches HNR, the 3 R34-unit experiment better captures the mass field profile above $750 \mathrm{mb}$ (Fig. 11b). The relative humidity fields in the low-level (LL, $850 \mathrm{mb}$ ), midlevel (ML, $500 \mathrm{mb}$ ), and upper-level (UL, $200 \mathrm{mb}$ ) environment corroborate this description (Figs. 12b and 12c). While both experiments produce wetter environments, the 3 R34-unit experiment exhibits the correct placement of the moist (north and east) and dry (south) ML and UL conditions. Neither experiment produces the dry center as is depicted in HNR (Fig. 12a). Both experiments accurately estimate the deep-layer $(200-850 \mathrm{mb}$ ) vertical wind shear magnitude ahead of the storm, but only the 3 R34-unit experiment correctly captures the direction of the shear vector as indicated by the vector overlaid on the analysis fields in Figs. 10 and 12. These shear values are valid for the environment $500 \mathrm{~km}$ ahead of the TC position, which indicates the conditions that the $\mathrm{TC}$ expects to encounter in about $24 \mathrm{~h}$, and not the current shear conditions. Overall, the 3 R34-unit experiment produces a more accurate analysis due to the vertical structure of the mass and kinematic fields.

The experiment using data spaced $40^{\circ}$ along the 1.5 R34-unit track struggles to intensify the TC at the same rate as the HNR intensifies. Therefore, cycles prior to the completion of rapid intensification dominate the intensity forecast error differences between experiments. Largest differences in the vortex region appear in the

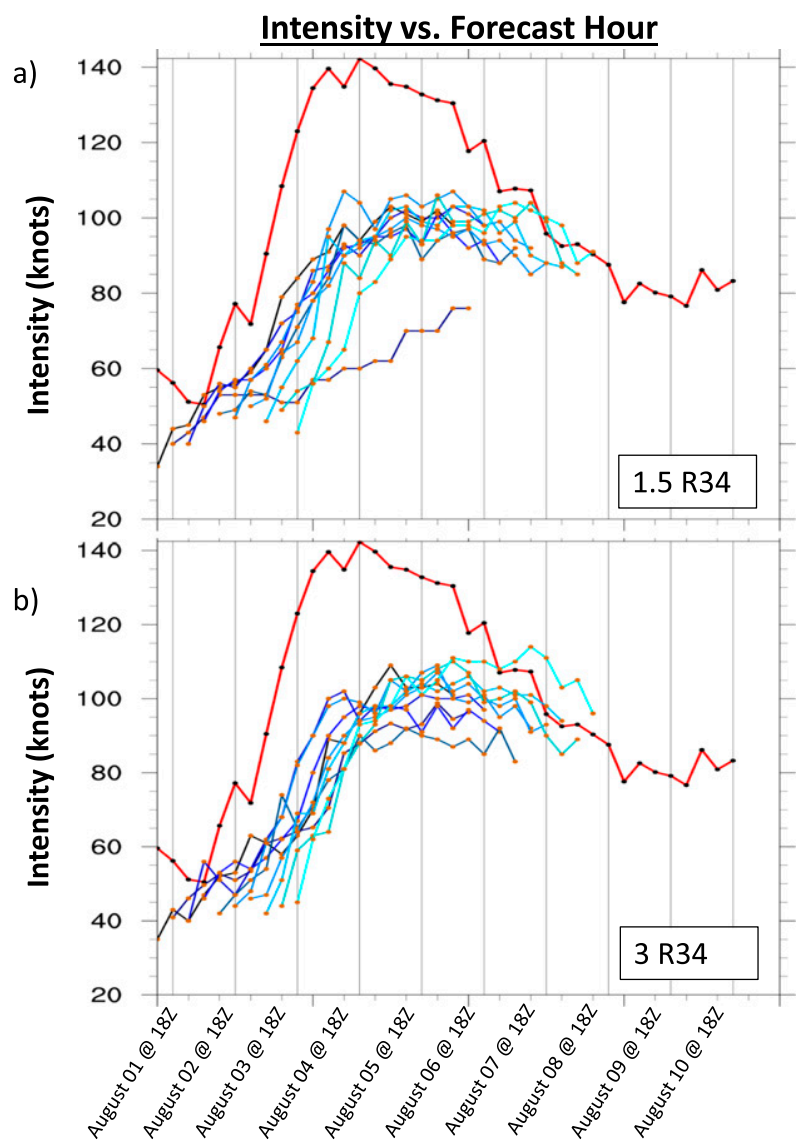

FIG. 9. Trend in maximum 10-m winds by cycle for the (a) 1.5 R34-unit radial distance experiment and (b) 3 R34-unit radial distance experiment. Earlier cycles are dark blue and trend lighter for each subsequent cycle, and the red line indicates the maximum 10-m winds of the HNR.

midlevel moisture fields as depicted in the 24-h forecasts initialized during RI at 0000 UTC 3 August (Figs. 13b and 13c). Both experiments exhibit a larger, dry center than HNR (Fig. 13a), but the entrainment of drier air into the southeast side of the vortex in the 1.5 R34unit experiment disrupts the organization of the ML moisture field (Fig. 13b), potentially leading to the degradation in forecast. This experiment generally overmoistens the near-TC environment as displayed in the average profile (Fig. 11c) where the majority of the wetter conditions come from the south (Fig. 11d). In contrast, the experiment using dropsondes along the 3 R34-unit distance produces an average profile close to HNR. Differences in the ML moisture may cause the intensity forecast improvement to diminish after $60 \mathrm{~h}$. In the near-TC environment, the average ML profile is too dry, and the average ML profile south of the TC is too saturated as depicted in the skew $T-\log p$ diagrams in Fig. 11. However, the vortex is better protected from the ML dry air which may contribute to the better intensity 


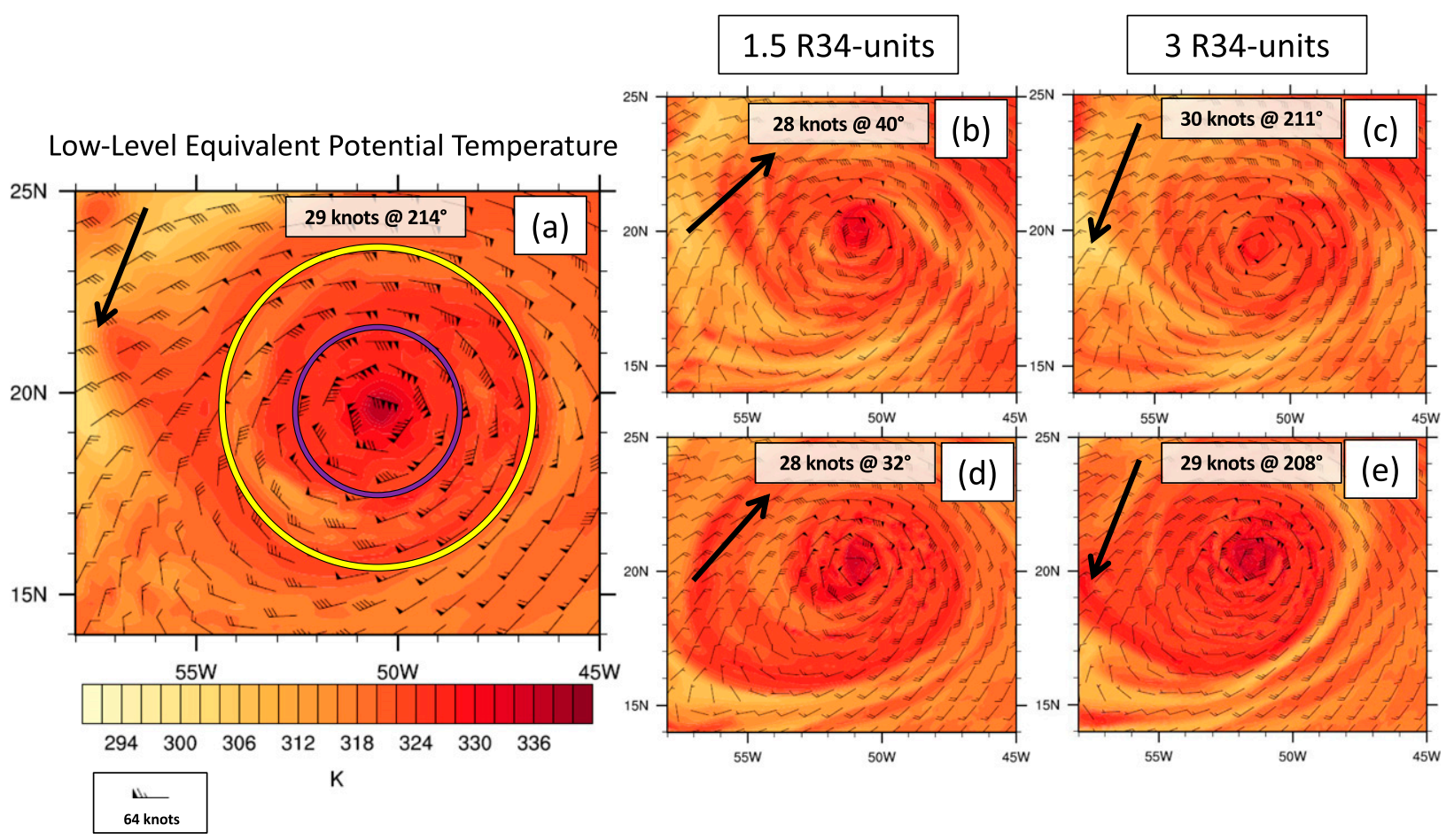

FIG. 10. 850-mb equivalent potential temperature field and wind barbs valid at 0000 UTC 4 Aug for (a) HNR, (b) analysis field from 1.5 R34-unit experiment using $40^{\circ}$ dropsonde spacing, (c) analysis field from 3 R34-unit experiment using 20 dropsonde spacing, (d) 24-h forecast field initialized at 0000 UTC 3 Aug from 1.5 R34-unit experiment using $40^{\circ}$ dropsonde spacing, and (e) 24-h forecast field initialized at 0000 UTC 3 Aug from 3 R34-unit experiment using $20^{\circ}$ dropsonde spacing. The black arrow in the top left represents the deep-layer vertical wind shear 300-700 km ahead of each field's TC. Range rings in (a) represent the radial distance of the G-IV flight track for the 1.5 R34-unit radial distance experiment (purple) and 3 R34-unit radial distance experiment (yellow).

forecast in the short-term. This comparison can be visualized in the RH 24-h forecast fields shown in Figs. 13b and 13c. Just as in the analysis fields for these two experiments, the LL temperature is more consolidated near the core (Figs. 10d and 10e), and the direction of deep-layer environment vertical wind shear is accurately produced by the 3 R34-unit experiment, as specified by the vector overlaid on each of the forecast fields. In addition to producing a better analysis, the 3 R34-unit experiment yields a more accurate forecast due to an improved moisture and kinematic structure. This result supports the average error statistics described above and displayed in Fig. 4c. The results suggest that consistently sampling in the near-TC environment has a positive impact on the intensity forecast, shown by the flight track locations relative to each nature run analysis field (Figs. 10a, 12a, and 13a). This improvement has the potential to affect the track forecast given a better depiction of the long-range synoptic-scale environment.

\section{Conclusions}

AOML's high-resolution regional OSSE system provided a novel framework for testing improvements to current hurricane targeting techniques. An evaluation of the circumnavigation portion of NHC's current targeting procedure has been evaluated, and this method proves to be beneficial for hurricane forecasting. Potential improvements to the procedure were examined using dropsonde distribution sensitivity experiments. The results of these experiments suggest using the size of the target TC to determine dropsonde locations. This ensures that the observations collected consistently sample the conditions in the near-storm environment regardless of natural size increases through time. The sensitivity of storm-relative dropsonde locations on hurricane track and intensity prediction was assessed in both radial and azimuthal contexts. These experiments compared the relative impact of uniform dropsonde configurations on the forecasts and analyses of a Hurricane nature run. All experiments were performed using a modified version of the operational HWRF Model.

Assimilating G-IV dropsonde data in the near-TC environment produced a positive impact on both track and intensity forecasts within the 2-3-day range. The addition of G-IV data improved the short-term track forecast of HNR by improving the synoptic-scale 


\section{Experiment Analyses}
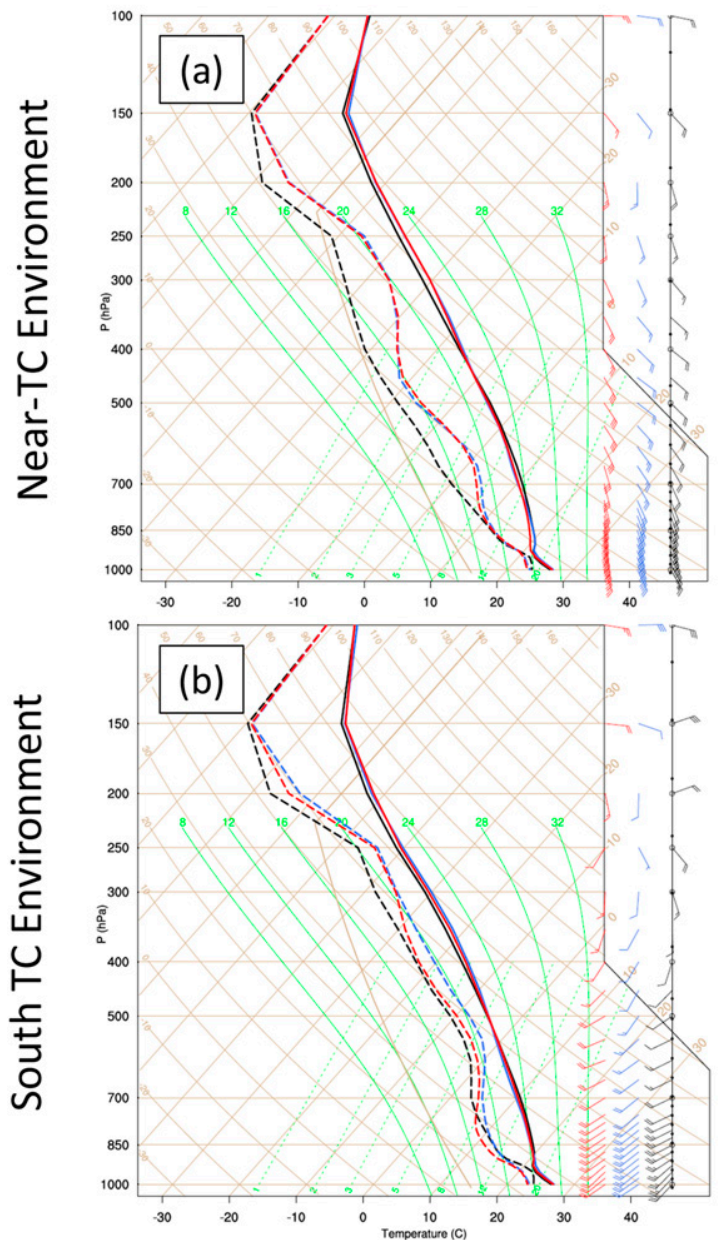

Experiment Forecasts
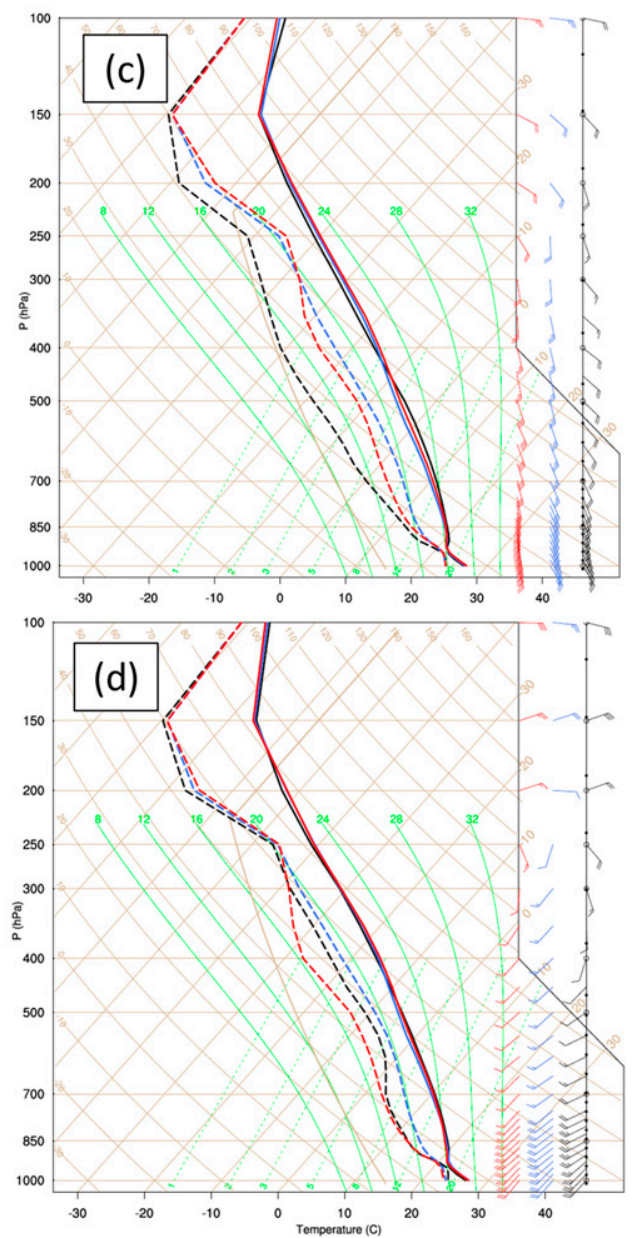

FIG. 11. Skew $T-\log p$ graph of (a),(c) the near-TC environment and (b),(d) the environment south of the TC comparing the HNR (black) to 1.5 R34-unit experiment using $40^{\circ}$ dropsonde spacing (blue) and 3 R34-unit experiment using $20^{\circ}$ dropsonde spacing (red). Comparisons of experiment analyses at 0000 UTC 4 Aug are shown in (a),(b) and experiment 24-h forecasts initialized at 0000 UTC 3 Aug are shown in (c),(d). Solid lines represent the temperature curve and dashed lines represent the dewpoint curve for each model field.

environment in all experiments, with its best forecast produced by the experiment where dropsondes were deployed every $40^{\circ}$ along a G-IV path at a distance of 1.5 R34 units from the center. Long-term forecast errors were dominated by discrepancies in the approaching front from the west (not shown), which may be worsened by the overfitting of observations known to be an issue for 3Dvar schemes. This experiment improved the subtropical ridge characteristics to the north, but also negatively impacted the TC strength. In weakening and shrinking the vortex, the mean flow of the environment wind field did not provide much forcing for TC motion. These factors contributed to the improvement of the short-term track forecast, albeit for the wrong reasons and at the expense of an accurate intensity estimate. A better analysis of the vortex structure may extend track improvements by allowing the potential TC to follow the corrected environmental flow given by these data. In the long-range, track forecasts will likely depend on the timing and strength of the approaching front, which exists outside of the current HWRF domain.

Generally, this data did not improve the ability for HWRF to capture rapid intensification. However, in one experiment where dropsondes were deployed every $20^{\circ}$ along a G-IV flight track located 3 R34 units from the center, rapid intensification was well-produced despite a time offset due to inherent limitations of the 3D-Var data assimilation system. The experiment also generated a significant positive impact on the intensity 
(a) Nature Run
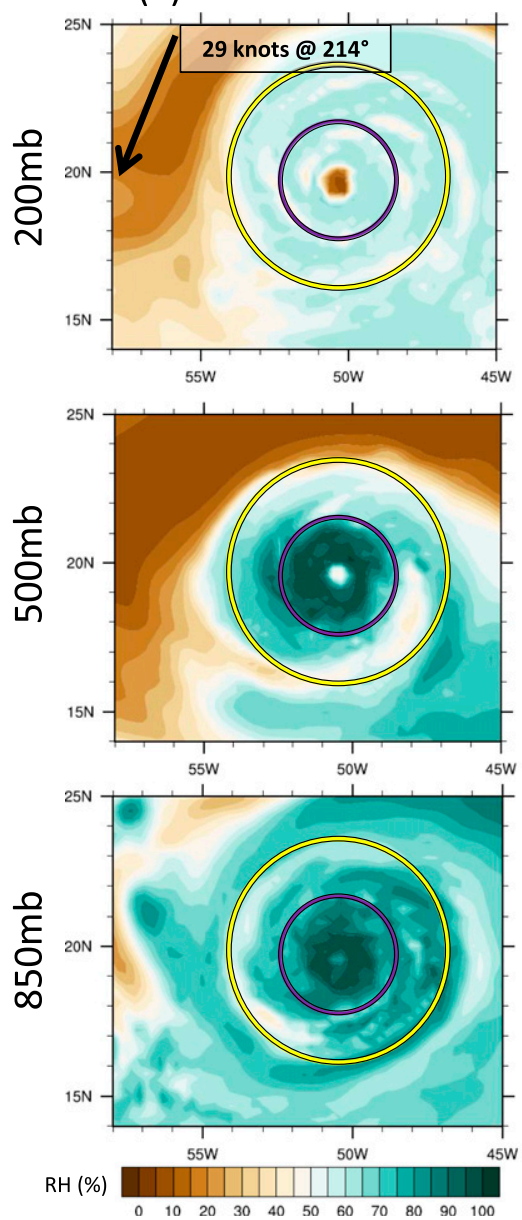

(b) 1.5 R34 units
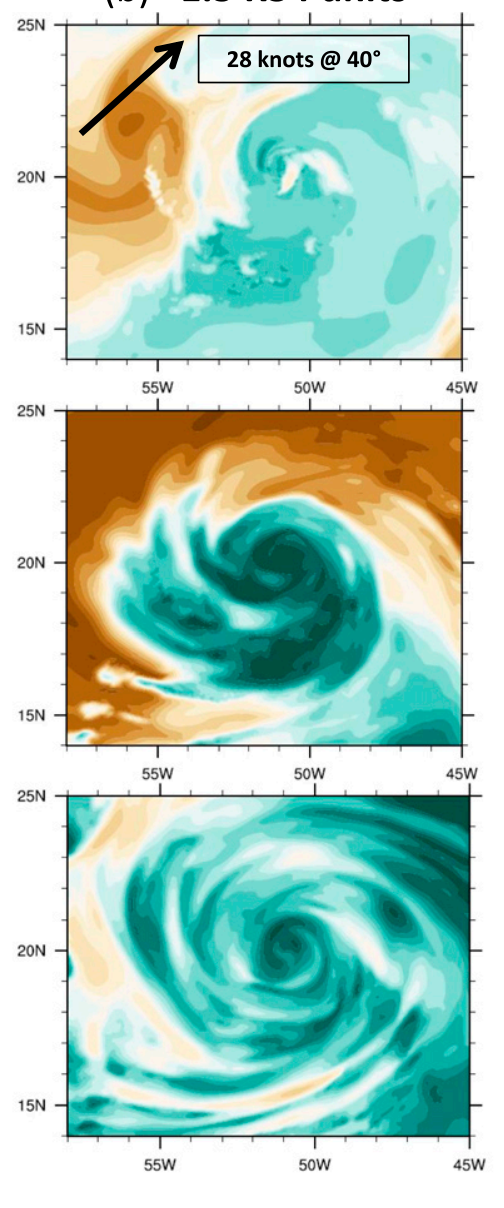

(c) 3 R34 units
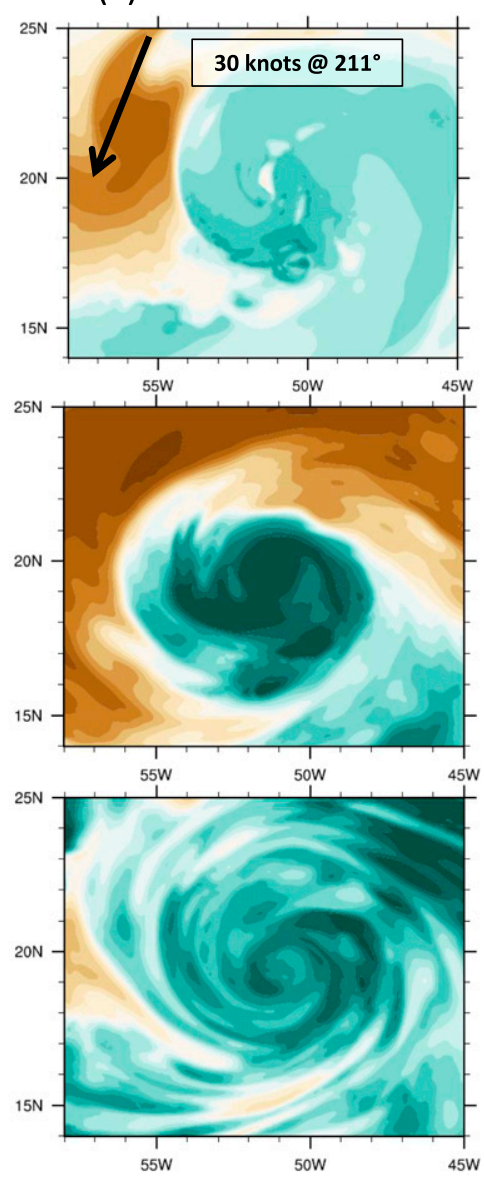

FIG. 12. (a) Upper-level, midlevel, and lower-level relative humidity fields for HNR. The black vector in the top left of the top plot represents the deep-layer vertical wind shear 300-700 km ahead of HNR. The purple circle represents the radial distance of the G-IV flight track for the 1.5 R34-unit radial distance experiment, and the yellow circle represents the radial distance of the G-IV flight track for the 3 R34-unit radial distance experiment. (b) Upper-level, midlevel, and lower-level relative humidity analysis fields for the 1.5 R34-unit experiment with $40^{\circ}$ dropsonde spacing. The black vector in the top left of the top plot represents the deep-layer vertical wind shear 300-700 km ahead of the TC. (c) Upper-level, midlevel, and lower-level relative humidity analysis fields for the 3 R34-unit experiment with $20^{\circ}$ dropsonde spacing. The black vector in the top left of the top plot represents the deep-layer vertical wind shear $300-700 \mathrm{~km}$ ahead of the TC.

forecast for more than 2 days. This may be a result of a better representation of the near-storm conditions affecting the initial vortex. In fact, this experiment yielded the best analysis and forecast TC structure of any other experiment due to the more accurate vertical structure of the mass and momentum fields in the near-TC environment. Overall, differences in intensity forecasts among experiments are dominated by the asymmetry and position of the vortex within the synoptic-scale wind field. In improving the vortex characteristics, the track forecast was slightly degraded due to a stronger steering effect in the short-term, thus adjusting the motion farther to the north and causing an early recurvature in the long-range track forecast. The relative track forecast degradation is not statistically significant when compared to other experiments.

It is important to note that given the nature of these regional sensitivity OSSEs, additional hurricane nature runs would be required to produce more robust error statistics. Since the sample size is restricted to just one hurricane, these results cannot be generalized to all TCs. The purpose of these results is to provide a quantification of the potential for improvement on NHC's existing targeting software by evaluating various configurations in dropsonde distribution. The results from these experiments suggest two alterations to NHC's G-IV surveillance software: 1) consistent sampling of the near-TC environment by using the radius of 34-kt winds 
(a) Nature Run
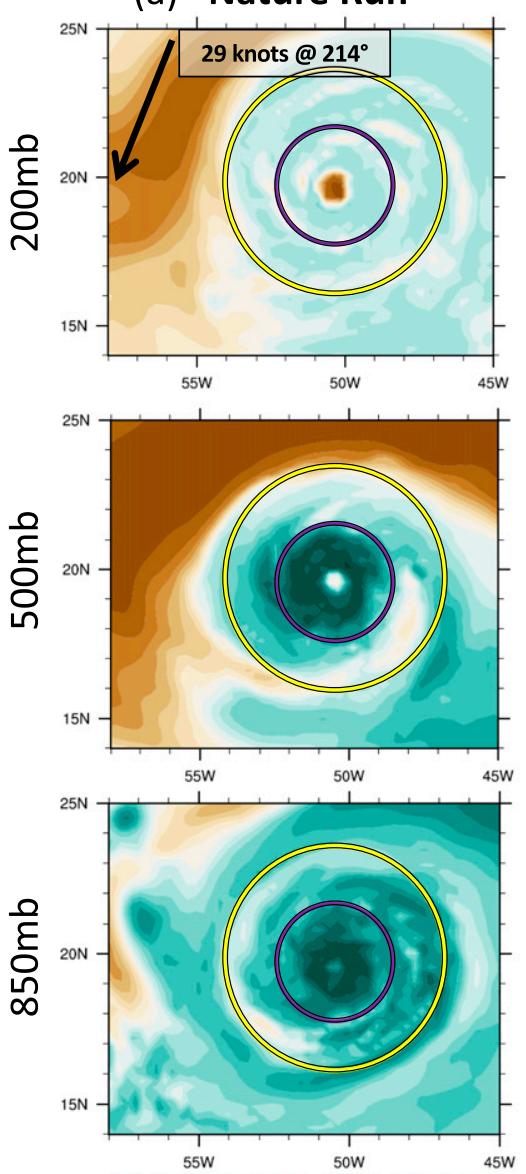

$\mathrm{RH}(\%)$ (b) 1.5 R34 units
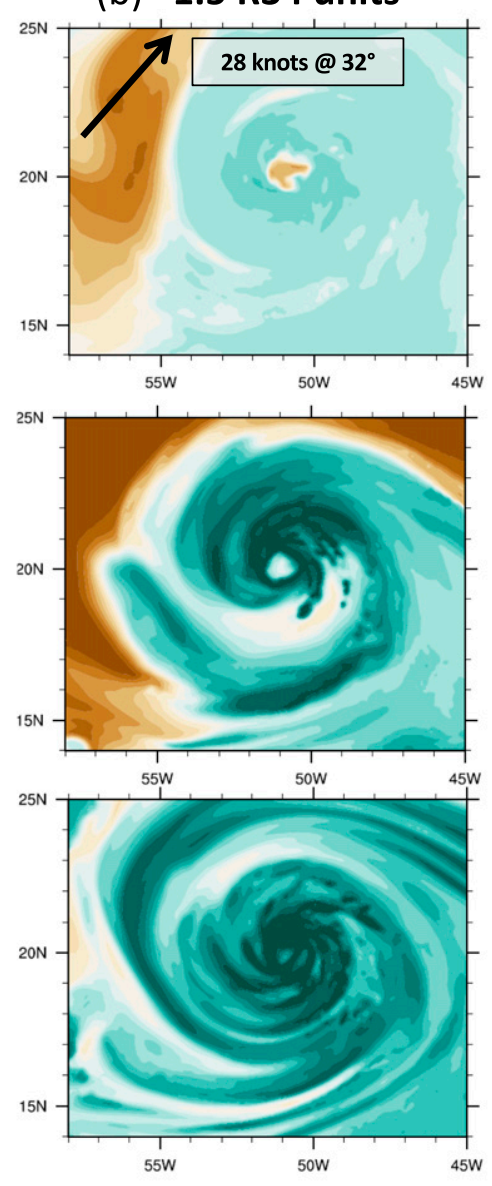

(c) 3 R34 units
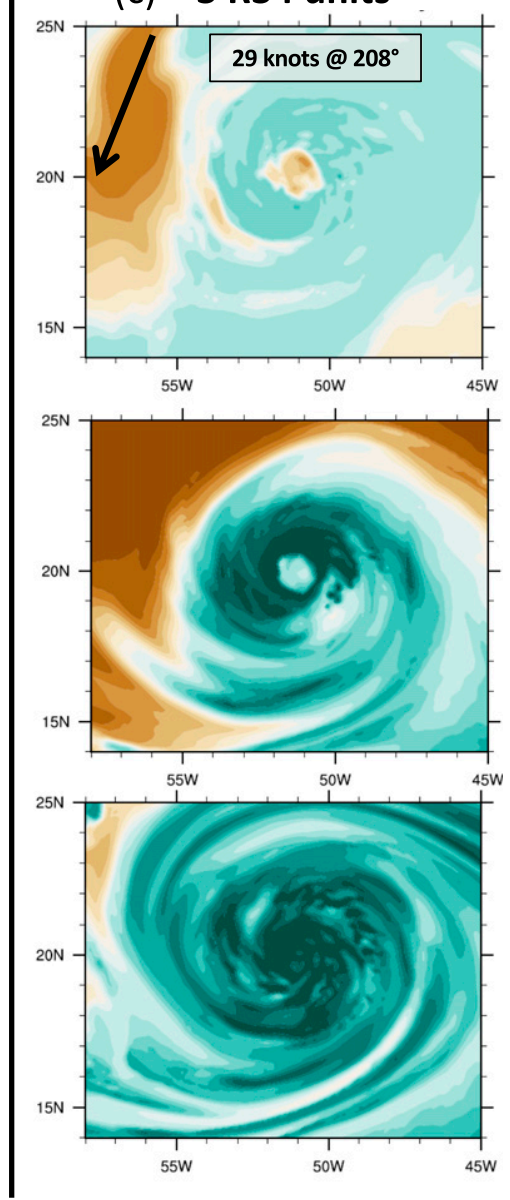

FIG. 13. As in Fig. 12, but (b) and (c) are showing 24-h forecast fields instead of analyses.

to normalize G-IV dropsonde deployment locations and 2) collecting observations in regions of shear or moisture suspected to affect the development and intensification of a potential TC target. The relative impacts generated in this regional OSSE framework show promise in guiding prospective improvements to targeting techniques expected to be implemented for operational use.

Additional G-IV dropsonde sensitivity experiments can include testing different flight patterns to replace the standard circumnavigation path and exploring ensemble sampling strategies that target regions of high uncertainty in various variable fields. Future studies aim to mitigate some of the limitations described in section 3 . The impact that observations have on an analysis is highly dependent on the data assimilation scheme used to incorporate those measurements. As an upgrade to AOML's regional OSSE framework, the software will include the flexibility of more data assimilation systems, including the hybrid 3D-Var-ensemble Kalman filter method used in many operational models. This allows for possible enhancements of the impacts seen above by reducing the likelihood of imbalances due to assumptions in defining the error covariance matrix in a pure 3D-Var method. Radiance assimilation problems known to affect regional models will be avoided by increasing the model top and removing the bias correction typically used in the operational system. Finally, to address the sample size limitation, a new basin-scale nature run is currently being validated. In addition to adding more cases to the sample, this innovative regional nature run includes multiple hurricanes which are all integrated at high resolution, so storm-to-storm interaction is enhanced. This large, uniform domain consists of both the North Atlantic and east Pacific basins and can be used for simulating measurements in the distant environment crucial for long-term hurricane track forecasts.

Acknowledgments. This work was supported by the Quantitative Observing System Assessment Program 
(QOSAP). We acknowledge AOML's Hurricane Research Division scientists Jason Sippel, Robert Rogers, Frank Marks, Jason Dunion, and Sim Aberson for valued discussions, and whose expertise bridged the gap between tropical cyclone science and operations. We greatly appreciate the direction and feedback given by the National Hurricane Center, especially Chris Landsea, Michael Brennan, and Mark DeMaria. We also thank the editor, Ryan Torn, for his valuable suggestions to improve this paper.

\section{REFERENCES}

Aberson, S. D., 2002: Two years of operational hurricane synoptic surveillance. Wea. Forecasting, 17, 1101-1110, https://doi.org/ 10.1175/1520-0434(2002)017<1101:TYOOHS>2.0.CO;2.

_ 2003: Targeted observations to improve operational tropical cyclone track forecast guidance. Mon. Wea. Rev., 131, 16131628, https://doi.org/10.1175//2550.1.

— tensity forecasts of GPS dropwindsonde observations from the first-season flights of the NOAA Gulfstream-IV jet aircraft. Bull. Amer. Meteor. Soc., 80, 421-427, https://doi.org/10.1175/ 1520-0477(1999)080<0421:IOHTAI >2.0.CO;2.

_, S. J. Majumdar, C. A. Reynolds, and B. J. Etherton, 2011: An observing system experiment for tropical cyclone targeting techniques using the Global Forecast System. Mon. Wea. Rev., 139, 895-907, https://doi.org/10.1175/2010MWR3397.1.

Atlas, R., 1997: Atmospheric observations and experiments to assess their usefulness in data assimilation. J. Meteor. Soc. Japan, 75, 111-130, https://doi.org/10.2151/jmsj1965.75.1B_111.

— L. Bucci, B. Annane, A. Aksoy, S. Murillo, J. Delgado, S. J. Majumdar, and L. Cucurull, 2015a: Observing System Simulation Experiments to assess the potential impact of new observing systems on hurricane forecasting. Mar. Technol. Soc. J., 49, 140-148, https://doi.org/10.4031/MTSJ.49.6.3.

— ments (OSSEs) to evaluate the potential impact of an Optical Autocovariance Wind Lidar (OAWL) on numerical weather prediction. J. Atmos. Oceanic Technol., 32, 1593-1613, https:// doi.org/10.1175/JTECH-D-15-0038.1.

Bell, G. D., and M. Chelliah, 2006: Leading Tropical modes associated with interannual and multidecadal fluctuations in North Atlantic hurricane activity. J. Climate, 19, 590-612, https://doi.org/10.1175/JCLI3659.1.

Bishop, C. H., and Z. Toth, 1999: Ensemble transformation and adaptive observations. J. Atmos. Sci., 56, 1748-1765, https:// doi.org/10.1175/1520-0469(1999)056<1748:ETAAO>2.0.CO;2.

— B. J. Etherton, and S. J. Majumdar, 2001: Adaptive sampling with the ensemble Transform Kalman Filter. Part I: Theoretical Aspects. Mon. Wea. Rev., 129, 420-436, https:// doi.org/10.1175/1520-0493(2001)129<0420:ASWTET>2.0.CO;2.

Burpee, R. W., J. L. Franklin, S. J. Lord, R. E. Tuleya, and S. D. Aberson, 1996: The impact of Omega dropwindsondes on operational hurricane track forecast models. Bull. Amer. Meteor. Soc., 77, 925-993, https://doi.org/10.1175/15200477(1996)077<0925:TIOODO > 2.0.CO;2.

Daescu, D. N., and R. Todling, 2010: Adjoint sensitivity of the model forecast to data assimilation system error covariance parameters. Quart. J. Roy. Meteor. Soc., 136, 2000-2012, https://doi.org/10.1002/qj.693.
DeMaria, M., and J. Kaplan, 1994: A Statistical Hurricane Intensity Prediction Scheme (SHIPS) for the Atlantic basin. Wea. Forecasting, 9, 209-220, https://doi.org/10.1175/1520-0434(1994) 009<0209:ASHIPS > 2.0.CO;2.

—, and - 1999: An Updated Statistical Hurricane Intensity Prediction Scheme (SHIPS) for the Atlantic and eastern North Pacific basins. Wea. Forecasting, 14, 326-337, https:// doi.org/10.1175/1520-0434(1999)014<0326:AUSHIP>2.0.CO;2.

_-, M. Mainelli, L. K. Shay, J. A. Knaff, and J. Kaplan, 2005: Further improvements to the Statistical Hurricane Intensity Prediction Scheme (SHIPS). Wea. Forecasting, 20, 531-554, https://doi.org/10.1175/WAF862.1.

Elsberry, R. L., E. L. Weniger, and D. H. Meanor, 1988: A statistical tropical cyclone intensity forecast technique incorporating environmental wind and vertical wind shear information. Mon. Wea. Rev., 116, 2142-2154, https://doi.org/10.1175/15200493(1988) $116<2142$ :ASTCIF $>2.0$.CO;2.

Elsner, J. B., K. Liu, and B. Kocher, 2000: Spatial variations in major U.S. hurricane activity: Statistics and a physical mechanism. J. Climate, 13, 2293-2305, https://doi.org/10.1175/15200442(2000)013<2293:SVIMUS > 2.0.CO;2.

Evans, J. L., G. J. Holland, and R. L. Elsberry, 1991: Interactions between a barotropic vortex and an idealized subtropical ridge. Part I: Vortex motion. J. Atmos. Sci., 48, 301-314, https://doi.org/ 10.1175/1520-0469(1991)048<0301:IBABVA > 2.0.CO;2.

Gopalakrishnan, S. G., S. Goldenberg, T. Quirino, X. Zhang, F. Marks, K. Yeh, R. Atlas, and V. Tallapragada, 2012: Toward improving high-resolution numerical hurricane forecasting: Influence of model horizontal grid resolution, initialization, and physics. Wea. Forecasting, 27, 647-666, https://doi.org/10.1175/ WAF-D-11-00055.1.

Hendricks, E. A., M. S. Peng, and T. Li, 2013: Evaluation of multiple dynamic initialization schemes for tropical cyclone prediction. Mon. Wea. Rev., 141, 4028-4048, https://doi.org/ 10.1175/MWR-D-12-00329.1.

Hoffman, R. N., and R. Atlas, 2016: Future observing system simulation experiments. Bull. Amer. Meteor. Soc., 97, 16011616, https://doi.org/10.1175/BAMS-D-15-00200.1.

Landsea, C. W., G. D. Bell, W. M. Gray, and S. B. Goldenberg, 1998: The extremely active 1995 Atlantic hurricane season: Environmental conditions and verification of seasonal forecasts. Mon. Wea. Rev., 126, 1174-1193, https://doi.org/10.1175/ 1520-0493(1998)126<1174:TEAAHS > 2.0.CO;2.

Liou, K. N., and M. D. Chou, 2008: Recent Progress in Atmospheric Sciences: Applications to the Asia-Pacific Region. World Scientific, 496 pp.

Majumdar, S. J., S. D. Aberson, C. H. Bishop, R. Buizza, M. S. Peng, and C. A. Reynolds, 2006: A comparison of adaptive observing guidance for Atlantic tropical cyclones. Mon. Wea. Rev., 134, 2354-2372, https://doi.org/10.1175/MWR3193.1.

- M. J. Brennan, and K. Howard, 2013: The impact of dropwindsonde and supplemental rawindsonde observations on track forecasts for Hurricane Irene (2011). Wea. Forecasting, 28, 1385-1403, https://doi.org/10.1175/WAF-D-13-00018.1.

_ , and Coauthors, 2011: Targeting observations for improving numerical weather prediction: An overview. WMO/THORPEX Article 15, WMO, 45 pp., https://www.wmo.int/pages/prog/arep/ wwrp/new/documents/THORPEX_No_15.pdf.

McNoldy, B., B. Annane, S. Majumdar, J. Delgado, L. Bucci, and R. Atlas, 2017: Impact of assimilating CYGNSS data on tropical cyclone analyses and forecasts in a regional OSSE framework. Mar. Tech. Soc. J., 51, 7-15, https://doi.org/10.4031/ MTSJ.51.1.1. 
Nolan, D. S., R. Atlas, K. T. Bhatia, and L. R. Bucci, 2013: Development and validation of a hurricane nature run using the joint OSSE nature run and the WRF model. J. Adv. Model. Earth Syst., 5, 382-405, https://doi.org/10.1002/jame.20031.

Peng, M. S., and C. A. Reynolds, 2006: Sensitivity of tropical cyclone forecasts as revealed by singular vectors. J. Atmos. Sci., 63, 2508-2528, https://doi.org/10.1175/JAS3777.1.

Reale, O., J. Terry, M. Masutani, E. Andersson, L. P. Riishojgaard, and J. C. Jusem, 2007: Preliminary evaluation of the European Centre for Medium-Range Weather Forecasts' (ECMWF) Nature Run over the tropical Atlantic and African monsoon region. Geophys. Res. Lett., 34, L22810, https://doi.org/ 10.1029/2007GL031640.

Reynolds, C. A., M. S. Peng, S. J. Majumdar, S. D. Aberson, C. H. Bishop, and R. Buizza, 2007: Interpretation of adaptive observing guidance for Atlantic tropical cyclones. Mon. Wea. Rev., 135, 4006-4029, https://doi.org/10.1175/2007MWR2027.1.

Riishojgaard, L. P., Z. Ma, M. Masutani, J. S. Woollen, G. D. Emmitt, S. A. Wood, and S. Greco, 2012: Observation system simulation experiments for a global wind observing sounder. Geophys. Res. Lett., 39, L17805, https://doi.org/10.1029/2012GL051814.

Ryan, K., R. Atlas, L. Bucci, J. Delgado, B. Annane, and S. Murillo, 2016: OSSE assessment of a hyperspectral sounder and its potential impact on hurricane forecasts. 20th Conf. on Integrated Observing and Assimilation Systems for the Atmosphere, Oceans, and Land Surface (IOAS-AOLS), New Orleans, LA, Amer. Meteor. Soc., 245, https://ams.confex.com/ams/ 96Annual/webprogram/Paper287953.html.

Sellwood, K. J., S. J. Majumdar, B. E. Mapes, and I. Szunyogh, 2008: Predicting the influence of observations on mediumrange forecasts of atmospheric flow. Quart. J. Roy. Meteor. Soc., 134, 2011-2027, https://doi.org/10.1002/qj.341.

Tallapragada, and Coauthors, 2015: Hurricane Weather Research and Forecasting (HWRF) Model: 2015 Scientific
Documentation. NCAR Tech. Note NCAR/TN-522+STR, 122 pp., https://doi.org/10.5065/D6ZP44B5.

Torn, R. D., 2014: The impact of targeted dropwindsonde observations on tropical cyclone intensity forecasts of four weak systems during PREDICT. Mon. Wea. Rev., 142, 2860-2878, https://doi.org/10.1175/MWR-D-13-00284.1.

- and G. J. Hakim, 2008: Ensemble-based sensitivity analysis. Mon. Wea. Rev., 136, 663-677, https://doi.org/10.1175/ 2007MWR2132.1.

$\longrightarrow$, and - 2009: Initial condition sensitivity of western Pacific extratropical transitions determined using ensemble-based sensitivity analysis. Mon. Wea. Rev., 137, 3388-3406, https:// doi.org/10.1175/2009MWR2879.1.

Wu, C.C., and Coauthors, 2009: Intercomparison of targeted observation guidance for tropical cyclones in the northwestern Pacific. Mon. Wea. Rev., 137, 2471-2492, https://doi.org/ 10.1175/2009MWR2762.1.

Xie, B., F. Zhang, Q. Zhang, J. Poterjoy, and Y. Weng, 2013: Observing strategy and observation targeting for tropical cyclones using ensemble-based sensitivity analysis and data assimilation. Mon. Wea. Rev., 141, 1437-1453, https://doi.org/ 10.1175/MWR-D-12-00188.1.

Zhang, F., and J. A. Sippel, 2009: Effects of moist convection on hurricane predictability. J. Atmos. Sci., 66, 1944-1961, https:// doi.org/10.1175/2009JAS2824.1.

Zhang, M., F. Zhang, X. Huang, and X. Zhang, 2011: Intercomparison of an ensemble Kalman filter with three- and four-dimensional variational data assimilation methods in a limited-area model over the month of June 2003. Mon. Wea. Rev., 139, 566-572, https://oi.org/10.1175/2010MWR3610.1.

Zhu, T., F. Weng, M. Masutani, and J. S. Woollen, 2012: Synthetic radiance simulation and evaluation for a Joint Observing System Simulation Experiment. J. Geophys. Res., 117, D23111, https:// doi.org/10.1029/2012JD017697. 